\title{
Determination of All Stabilizing Fractional-Order PID Controllers
}

\author{
Yung K. Lee \\ Faculty: John M. Watkins \\ Department of Electrical Engineering and Computer Science
}

\begin{abstract}
A novel method for finding all fractional-order (FO) proportional-integral-derivative (PID) controllers that stabilize a given system of integer or non-integer order is proposed. The stability bounds of such FO PID controllers are calculated in the frequency domain and are given in terms of the proportional gain $K_{p}$, integral gain $K_{i}$ and derivative gain $K_{d}$. In this paper, they will be plotted on the $\left(K_{p}, K_{i}\right)$ plane. A key advantage of this approach is that it provides the stability boundaries even when the transfer function of a system is not available, as long as the frequency response of the system can be obtained. An example is presented to illustrate the effectiveness of this method.
\end{abstract}

\section{Introduction}

Though PID controllers are clearly in the mainstream of the controls area, their non-integer order counterparts, so called $\mathrm{PI}^{\lambda} \mathrm{D}^{\mu}$ controllers (where $\lambda$ and $\mu$ are arbitrary real numbers) are receiving considerable attention. While integer-order (IO) mathematical models are easier to work with, real physical systems are often described more accurately through non-integer order modeling. In [1], a torsional system consisting of a rigid disk and a flexible shaft attached thereto is modeled using an FO transfer function. The resulting frequency response shows that the mechanical resonance effect is represented more naturally with an FO model than an IO model.

As in the case of IO PID controllers, the stability boundary of an FO PID controller is an important research topic and has received significant attention. In [2], the D-partition method, which has been used for the IO controller case, is used to find stability bounds of FO PI or $\mathrm{PI}^{\lambda}$ controllers for four cases. These cases include all the combinations of an IO/FO plant and an IO/FO controller. However, only a first order IO plant or an FO plant with the order $\alpha$ (where $0<\alpha<1)$ is used in [2]. In addition, Matignon's stability theorem is used to find the stability of an FO system [3].

Nonetheless, there still exists a need for more efficient and less complicated ways of finding all controllers that stabilize a given system with an IO or FO transfer function. In [4], the frequency response is used to find all stabilizing IO controllers for a given plant transfer function of an arbitrary IO. The stabilizing controllers that lie within the stability regions are plotted in three different planes: $\left(K_{p}, K_{i}\right)$, $\left(K_{p}, K_{d}\right)$ and $\left(K_{d}, K_{i}\right)$.

In this paper, a new method, similar to the one described in [4], is used to find all the stabilizing $\mathrm{PI}^{\alpha} \mathrm{D}^{\beta}$ controllers for a given plant transfer function using the frequency response. Because of limited space, results presented in this paper are limited to the $\left(K_{p}, K_{i}\right)$ plane for a fixed $K_{p}$ value. The results and an example will be described in section 2 .

\section{Determination of Stabilizing Controllers.}

Consider the following unity feedback control system shown in Fig. 1.

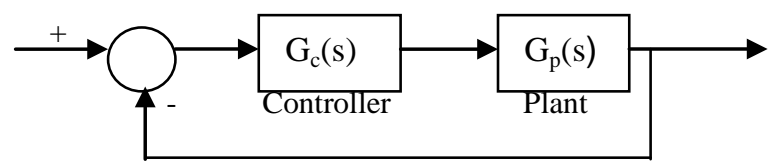

Fig. 1. Control system with negative unity feedback

The plant transfer function is $\mathrm{G}_{\mathrm{p}}(\mathrm{s})$. The $\mathrm{PI}^{\alpha} \mathrm{D}^{\beta}$ controller $\mathrm{G}_{\mathrm{c}}(\mathrm{s})$ is given by

$$
G_{c}(s)=K_{p}+\frac{K_{i}}{s^{\alpha}}+K_{d} s^{\beta}
$$

where $K_{p}, K_{i}$ and $K_{d}$ denote the proportional, integral and derivative gains, respectively, and $\alpha$ and $\beta$ are arbitrary positive real numbers.

\subsection{General Solution in $\left(K_{p}, K_{i}\right)$ Plane}

To determine the $K_{p}$ and $K_{i}$ values that stabilize the given transfer function, the characteristic equation in frequency domain is found by replacing $s$ with $j \omega$ in the closed-loop transfer function of Fig. 1, which is given by

$$
\Delta(j \omega)=1+G_{p}(j \omega) G_{c}(j \omega)
$$

The plant transfer function can be decomposed into real and imaginary parts as follows: 


$$
G_{p}(j \omega)=R_{p}(j \omega)+j I_{p}(j \omega)
$$

Setting (1-2) to zero and expanding it into real and imaginary parts gives us two equations and three unknowns: $K_{p}, K_{i}$ and $K_{d}$. We will fix the value of $K_{d}$ and find the stability region in the $(K p, K i)$ plane.

Solving these two equations for $\omega \neq 0$ and $\alpha \neq 2 n$ where $n$ is an interger, $K_{p}$ and $K_{i}$ are given by

$$
\begin{gathered}
K_{p}=-K_{d} \omega^{\beta} \frac{\sin \left(\frac{\pi}{2}(\alpha+\beta)\right)}{\sin \left(\frac{\pi}{2} \alpha\right)}-\frac{R_{p}(\omega)-\cot \left(\frac{\pi}{2} \alpha\right) I_{p}(\omega)}{\mid G_{p}(j \omega)^{2}} \\
K_{i}=K_{d} \omega^{\alpha+\beta} \frac{\sin \left(\frac{\pi}{2} \beta\right)}{\sin \left(\frac{\pi}{2} \alpha\right)}-\frac{\omega^{\alpha} I_{p}(\omega)}{\sin \left(\frac{\pi}{2} \alpha\right) \cdot\left|G_{p}(j \omega)\right|^{2}}
\end{gathered}
$$

where

$$
\left|G_{p}(j \omega)\right|^{2}=R_{p}^{2}(\omega)+I_{p}^{2}(\omega)
$$

\subsection{Example}

Consider the following plant with a second order transfer function having a time delay of 0.6 seconds

$$
G_{p}(s)=\frac{4-2 s}{s^{2}+s+9} e^{-0.6 s}
$$

which is described in [5] without the time delay. The objective here is to compare the stability region of an FO PID controller with that of an IO PID controller, along with their step responses. In this example, an FO PID controller with $\alpha=0.85$ and $\beta=0.7$ is used for the controller transfer function in (1-1).

In Fig. 2, the stability regions of the IO and FO PID controllers are plotted in the $\left(K_{p}, K_{i}\right)$ plane for $K_{d}=0.4$. As can be seen, the FO PID controller provides a much wider stability region than the conventional IO PID controller. Two arbitrary controllers are chosen from the stability regions in Fig. 2, as marked on the plot. For the IO PID, $K_{p}=0.2137$ and $K_{i}=3.0548$, and for the FO PID, $K_{p}=-$ 0.8508 and $K_{i}=1.6221$.

Fig. 3 shows the corresponding closed-loop step responses with the above IO and FO PID controllers. In the example, the closed-loop system with the IO PID controller has the percent overshoot of P.O $=92.7 \%$ and the $2 \%$ settling time of $t_{s}=31.3$ seconds, while the closed-loop system with the FO PID has the P.O $=32.9 \%$ and $t_{s}=14.1$ seconds.

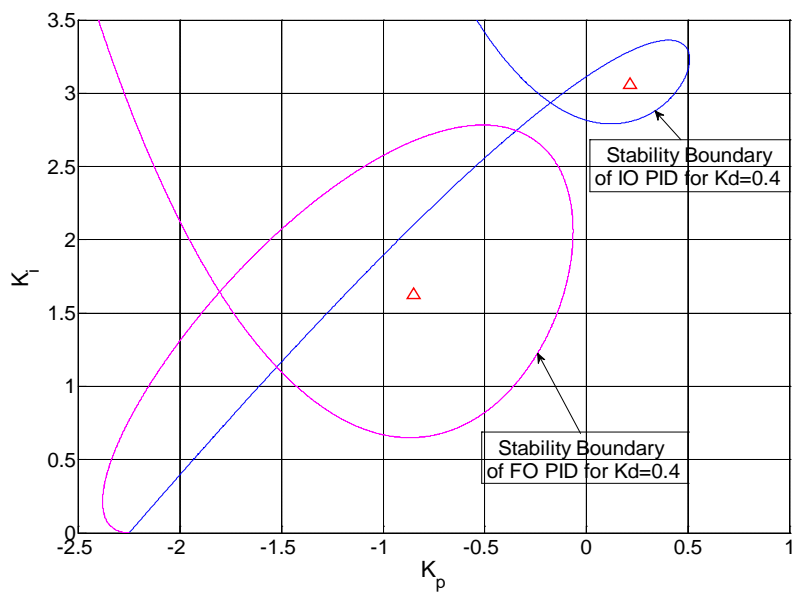

Fig. 2. Stability regions in $\left(K_{p}, K_{i}\right)$ plane for $K_{d}=0.4$

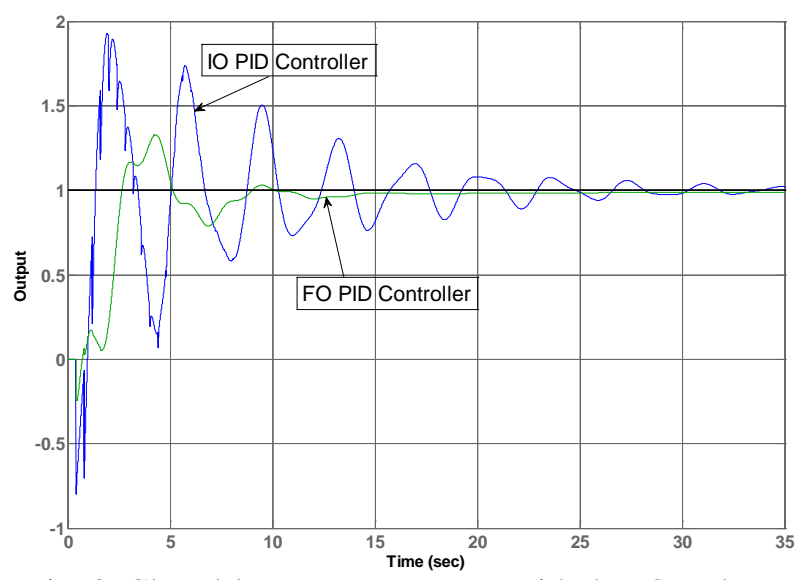

Fig. 3. Closed-loop step responses with the IO and FO PID controllers for $K_{d}=0.4$

\section{Conclusions}

As described in Section 2, a general solution for finding all FO stabilizing controllers for a given system transfer function of an arbitrary order is given in the $\left(K_{p}, K_{i}\right)$ plane for a fixed $K_{d}$ value.

\section{REFERENCES}

[1] S. Manabe, "A suggestion of fractional-order controller for flexible spacecraft attitude control,” Nonlinear Dynamics, vol. 29, no.1-4, pp.251-268, 2002.

[2] A. Ruszewski, "Stability regions of closed loop system with time delay inertial plant of fractional order and fractional order PI controller,” Bulletin of the Polish Academy of Sciences, Technical Sciences, vol. 56, No. 4, 2008.

[3] YangQuan Chen and Ivo Petráš and DingyuXue, "Fractional order control - a Tutorial," Proceedings of the 2009 American Control Conference, St. Louis, MO, USA, 2009.

[4] SeadSujoldžić and John M. Watkins, "Stabilization of an arbitrary order transfer function with time delay using a PID controller," Proceedings of the $45^{\text {th }}$ IEEE Conference on Decision and Control, San Diego, CA, USA, 2006.

[5] Gene F. Franklin, J. David Powell and Abbas Emami-Naeini, "Feedback control of dynamic systems," $4^{\text {th }}$ Ed., Prentice Hall, New Jersey, 2002. 